\title{
Effects of prolonged processing in standard and isotonic trichloroacetic acid (TCA) on cellular preservation in bone marrow trephines
}

\author{
R J Fitzmaurice, A Pitalia, A J Freemont
}

\begin{abstract}
The effects of a combined fixing and decalcifying solution, trichloroacetic acid, on prepared bone marrow specimens were evaluated to try to improve the speed of trephine biopsy processing in our laboratory. The morphological preservation obtained immediately after decalcification was complete was assessed, and also after prolonged immersion in each of two solutions. Trichloroacetic acid was made up in the standard manner using distilled water and in an isotonic form by the addition of sodium chloride. Prolonged immersion was performed to assess any possible adverse effects of this acidic solution.

By the time decalcification was complete, each solution gave morphological preservation sufficient for diagnostic purposes. However, after prolonged immersion, only the isotonic solution performed adequately.
\end{abstract}

Marrow trephine biopsy is a well established procedure for investigation of bone marrow disease. It is sometimes more informative than bone marrow aspirate as all cellular and stromal components of bone marrow may be examined and their spatial distribution assessed. ${ }^{1}$ Bone marrow aspirate smears may be diluted by peripheral blood resulting in falsely high numbers of polymorphs and lymphocytes, ${ }^{2}$ but there are also inherent sampling errors. ${ }^{34}$ On the other hand, the conventional method of processing trephine biopsy specimens-18 hours' formalin fixation and formic acid decalcification for 28 hours -is both more time consuming than the preparation of an aspirate smear, and sections lack the cytological detail of smears. Various measures to reduce processing time or to improve morphological preservation have proved to be a compromise. Plastic embedding, though yielding excellent morphology, is much more expensive than paraffin wax processing and requires special equipment, materials, and technical support. ${ }^{56}$ Frozen sections, although very rapid to prepare, do not provide good histological resolution, are rarely sufficiently thin, and are susceptible to crush and tear artefacts with resulting diffusion of antigen and its false localisation. $^{78}$

Trichloroacetic acid (TCA) is an acid coagulative fixative which has previously been suggested as a possible means of simultaneous fixation and decalcification. 9

\section{Methods}

To standardise the size of the test specimens and the cellularity of the contained bone marrow a femoral head, obtained at a hip replacement, was used as the source of samples. The head was hemi-sectioned and pieces of bone the same size as trephine biopsy specimens were obtained from uniformly red marrow.

Five per cent TCA ( $5 \mathrm{~g}$ TCA in $100 \mathrm{ml}$ distilled water) was used as a "standard" solution and an isotonic solution prepared by addition of $0.85 \mathrm{~g} \mathrm{NaCl}$ to $100 \mathrm{ml}$ of standard TCA. Prepared pieces of bone were placed into four universal bottles, two containing standard TCA and two containing isotonic TCA. To determine when decalcification was complete, bone from both types of TCA was $x$-rayed at hourly intervals. Once fully decalcified, the bone was removed from the TCA, washed thoroughly, and processed into wax on an automatic processor. Further pieces of bone were left in standard and isotonic TCA, respectively, for a full 80 hours and then processed as above. Sections ( $1 \mu \mathrm{m}$ and $4 \mu \mathrm{m})$ were cut on a rotary microtome and stained with haematoxylin and eosin.

\section{Results}

Decalcification in standard and in isotonic TCA took 11 and 12 hours, respectively. The pieces of bone left in standard and isotonic TCA for 80 hours were both decalcified after this period of time.

Examination of haematoxylin and eosin stained sections in respect of cellular morphology showed that treatment with standard and isotonic TCA gave comparable results after 11 and 12 hours, respectively (fig 1). Nuclei were exceptionally well preserved, the distinction between myeloid and erythroid cells being obvious. Stages of differentiation within cell lineages were also well demonstrated. However, after 80 hours of exposure to TCA, morphology was better preserved in the isotonic solution and had remained equivalent to that achieved immediately after decalcification. In contrast, in the sample processed through standard TCA, loss of nuclear definition and detail, and pronounced cytoplasmic deterioration were apparent (fig 2).

\section{Discussion}

Simultaneous fixation and decalcification in TCA is much faster than the usual formalin fixation/formic acid decalcification method 

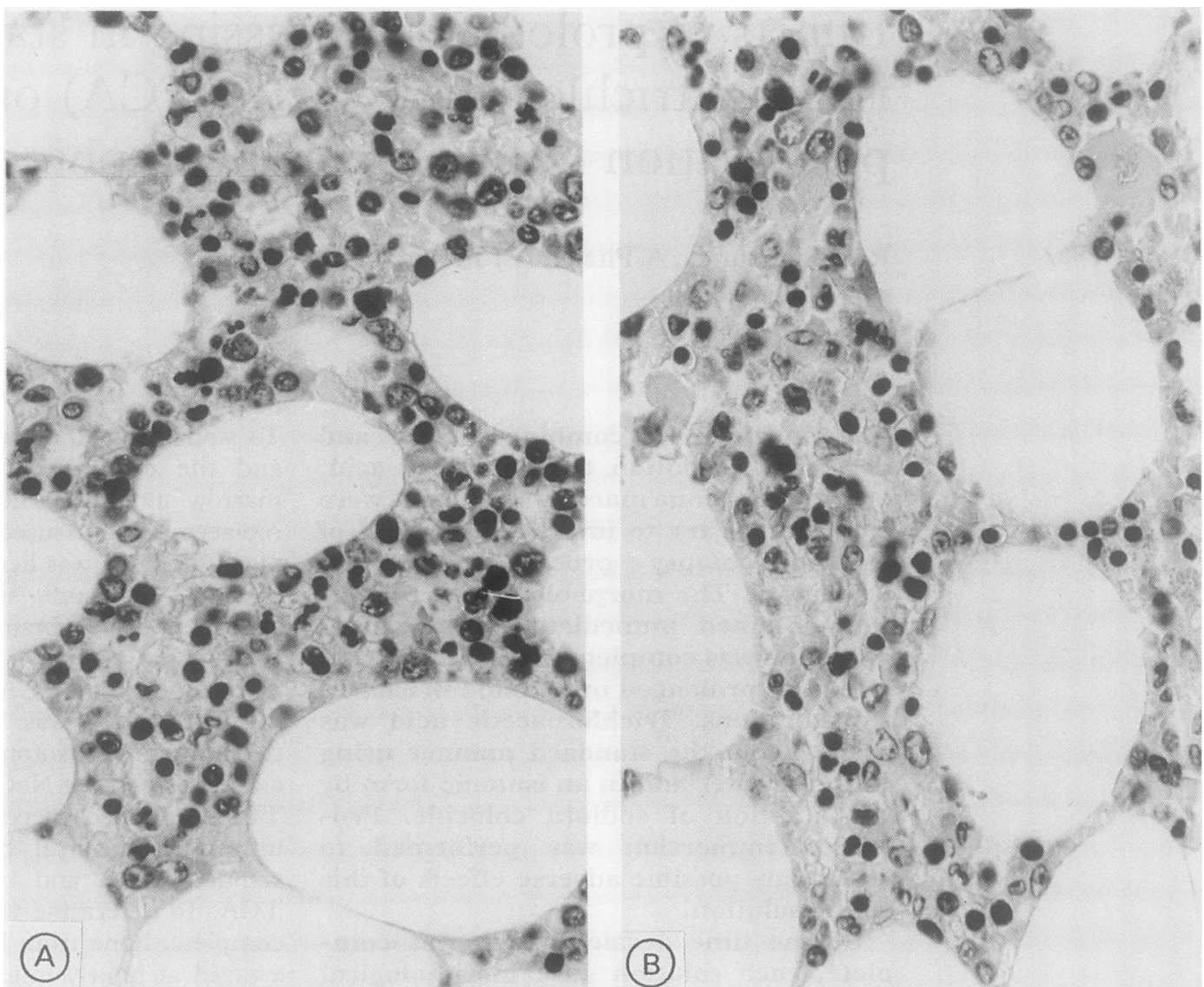

Figure 1 There is little apparent difference between fixation in standard TCA $(A)$ after 11 hours, and in isotonic TCA (B) after 12 hours (haematoxylin and eosin).

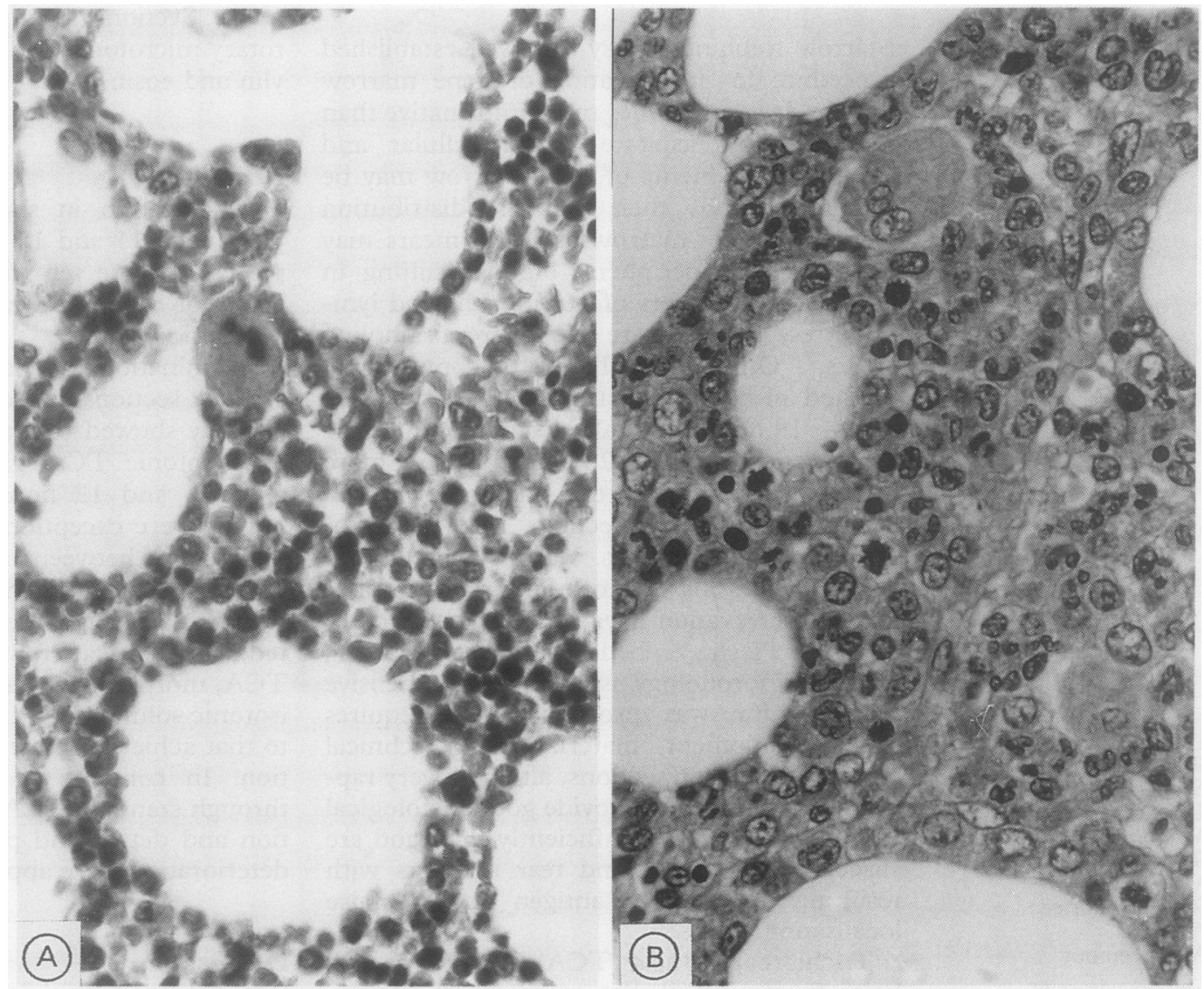

Figure 2 After 80 hours' fixation there is quite pronounced deterioration in cellular morphology in standard $(A)$ compared with isotonic (B) TCA. Detail remains good in isotonic TCA and myeloid granules are clearly seen (B) (haematoxylin and eosin). 
which takes a minimum of 46 hours. The implications of this rapid fixing/decalcifying protocol are that paraffin wax sections of a trephine biopsy specimen can be ready to examine the day after the biopsy specimen is taken. In practice, bone marrow trephine biopsy specimens can be processed after as little as three hours in isotonic TCA, with good results. However, to be certain of adequate fixation we stipulate a minimum fixation time of five hours.

Morphological preservation at 80 hours was better using isotonic TCA. Trichloroacetic acid is known to increase tissue volume, but the addition of $\mathrm{NaCl}$ to form an isotonic solution seems to reduce this effect. If there is a risk of excessive exposure to TCA, such as may occur if a biopsy specimen is left in TCA from Friday over the weekend to Monday, it is advisable to use an isotonic solution to prevent unwanted morphological deterioration.

Processing bone marrow trephine biopsy specimens in isotonic TCA not only cuts down on conventional processing time but also results in superior morphological preservation than that achievable using formalin fixation.
Shrinking artefact is reduced and nuclear detail enhanced thus more closely approximating the standard of plastic sections.

1 Bartl R, Frisch B, Burkhardt R. Introduction and structure and function of the normal bone marrow. In: Bone marrow biopsies revisited. $A$ new dimension for haematologic malignancies. 2nd edn. Basel: Karger, 1985:1-3, 9-15.

2 Holdrinet R, von Egmund J, Wessels J, Haanen C. A method for quantification of peripheral blood admixtures in bone marrow aspirates. Exp Haematol 1980;8:103-7.

3 Van der Valk P, Mullink H, Huijgens PC, Tadema TM, Vos W, Meijer CJLM. Immunohistochemistry in bone marrow diagnosis. Value of a panel of monoclonal antibodies on routinely processed bone marrow biopsies. Am $\mathcal{J}$ Surg Pathol 1989;13:97-106.

4 Wilkins BS, O'Brien CJ. Techniques for obtaining differential cell counts from bone marrow trephine biopsy specimens. f Clin Pathol 1988;41:558-61.

5 Islam A, Frisch B. Plastic embedding in routine histology I: Preparation of semithin sections of undecalcified marrow cores. Histopathology 1985;9:1263-74.

6 Anonymous. Plastic or paraffin? [Editorial.] Lancer 1989 ;i:139-40.

7 Bancroft JD, Stevens A. Frozen and related sections. In: Theory and practice of histological techniques. 2nd edn. Edinburgh: Churchill Livingstone, 1982:82-90.

8 Hall PA, Stearn PM, Butler MG, D'Ardenne AJD. Acetone/ periodate-lysine-paraformaldehyde (PLP) fixation and perioned morphology of cryostat (PLCion for immund improved morphology of cryostat sections for immur

Kiernan JA. Histological and histochemical methods: In: Theory and practice. Oxford: Pergamon Press, 1981:

\title{
Two techniques for electron opaque staining of elastic fibres using tannic acid in fresh and formalin fixed tissue
}

\author{
A Haidar, T A Ryder, M A Mobberley, J S Wigglesworth
}

\author{
Department of \\ Histopathology, Royal \\ Postgraduate Medical \\ School, Hammersmith \\ Hospital, Ducane \\ Road, London \\ W12 0HS \\ A Haidar \\ J S Wigglesworth \\ Electron microscopy \\ unit, Queen \\ Charlotte's and \\ Chelsea Hospital, \\ London \\ M A Mobberley \\ T A Ryder \\ Correspondence to: \\ Professor J S Wigglesworth \\ Accepted for publication \\ 29 November 1991
}

\begin{abstract}
Two electron microscopic staining techniques, one using tannic acid-glutaraldehyde as a fixative, and the other using tannic acid-uranyl acetate solution as a stain on ultra-thin sections of glutaraldehyde fixed material, were directly compared for elastic fibre staining on several human and animal tissues. Various concentrations of tannic acid were compared using both techniques. The two techniques were also compared on formalin fixed tissues.

The use of tannic acid-uranyl acetate solution as a stain on processed tissue is by far the more consistent technique and achieves equally good results on glutaraldehyde or formalin fixed tissue.

It is suggested that the use of the term tannic acid technique/method should be reserved for this particular method to achieve a meaningful interpretation of results in scientific papers.
\end{abstract}

Elastic fibres are components of the extracellular matrix and are present in most organs.
They are synthesised by connective tissue cells. ${ }^{1}$ Morphogenesis of normal and abnormal elastic fibres in adult and human fetal lung has recently been described using two different electron microscopic techniques ${ }^{23}$ involving the use of tannic acid, along with other methods. ${ }^{4-6}$ The exact mechanism involved in the interaction between tannic acid and elastin is unknown, although Hayat has pointed out some possible mechanisms. ${ }^{7}$ These include ionic interactions between carboxylate anions from tannic acid and cationic sites on the protein. Alternatively, hydrogen bonding may be involved. At an alkaline $\mathrm{pH}$ there may be binding of anionic oxygen to a cationic group such as ammonium in elastin. ${ }^{8}$ Kageyama and colleagues, ${ }^{9}$ using a modification of the original Kajikawa technique, ${ }^{2}$ have in fact shown that the maximum tannic acid staining of elastic fibres is in the $\mathrm{pH}$ range $7 \cdot 0-9 \cdot 0$.

The claim that tannic acid enhances staining of connective tissue elements including elastic fibres ${ }^{10}$ led us to compare these two tannic acid techniques $^{23}$ to see whether they could be used interchangeably. The two methods were applied to both formalin and glutaraldehyde fixed tissues. 